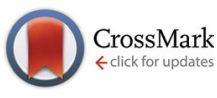

Cite this: Org. Biomol. Chem., 2015, 13, 2555

Received 5th December 2014,

Accepted 9th January 2015

DOI: 10.1039/c4ob02543a

www.rsc.org/obc

\section{A mechanistic proposal for the protodeboronation of neat boronic acids: boronic acid mediated reaction in the solid state $\uparrow$}

\author{
Gary Noonan $\star^{a}$ and Andrew G. Leach ${ }^{\text {b }}$
}

\begin{abstract}
A combined experimental and computational study suggests that a reduction in the entropy of activation in the solid state can lead to the protodeboronation of boronic acids.
\end{abstract}

Among the ways in which enzymes accelerate bimolecular reactions, one of the least contentious is that they organize the reacting molecules such that they are primed for reaction. ${ }^{1,2}$ Similar factors contribute to reaction acceleration by catalytic RNA and organic capsules. ${ }^{3}$ The entropic barrier to the reaction is reduced by the formation of stabilising interactions between the reactants and the catalyst; the reaction is rendered intramolecular. The formation of a crystalline lattice also involves molecular organization and if it arranges functional groups in such a way that they are primed for reaction, an accelerated reaction could result. Estimates of the entropic contribution to the free energy barrier for bimolecular reactions in solution at room temperature range from $2.5 \mathrm{kcal}$ $\mathrm{mol}^{-1}$ to $10.4 \mathrm{kcal} \mathrm{mol}^{-1} .{ }^{2,4}$ Removing even the low end of this range would amount to a large acceleration. As detailed below, we believe such an acceleration, caused by formation of the solid state, contributes to the protodeboronation of boronic acids.

Boronic acids are ubiquitous reagents in modern synthetic organic chemistry, due to their utility in Suzuki-Miyaura crosscoupling (SMCC) and many other synthetic transformations..$^{5-8}$ Most are stable reagents but certain examples (e.g. 2-heteroarylboronic acids) are susceptible to degradation via protodeboronation (PDeB), either under storage as the neat reagent, or under SMCC conditions. ${ }^{7,9-12}$ Some 'protected' derivatives have been developed to allow long-term storage and slow-

\footnotetext{
${ }^{a}$ AstraZeneca, Alderley Park, Macclesfield, SK10 4TG, UK.

E-mail: noonangary64@googlemail.com

${ }^{b}$ School of Pharmacy and Biomolecular Sciences, Liverpool John Moores University, James Parsons Building, Byrom Street, Liverpool, L3 3AF, UK.

E-mail: a.g.leach@ljmu.ac.uk

$\dagger$ Electronic supplementary information (ESI) available: All experimental and analytical information for compounds described, stability studies, computed geometries and energies, computational benchmarking, solid state parameters. See DOI: $10.1039 / \mathrm{c} 4 \mathrm{ob} 02543 \mathrm{a}$
}

release of delicate boronic acids under aqueous basic reaction conditions, ${ }^{9-15}$ but these reagents are less synthetically efficient and, for some, subtle changes in reaction conditions can affect their rates of release. ${ }^{13-15}$ By understanding PDeB we wished to enable the use of the more efficient parent boronic acids where possible.

Kuivila and co-workers performed extensive studies of PDeB under aqueous acid and base conditions and revealed mechanistic details of both. ${ }^{16-19}$ These are unlikely to be relevant here because boronic acids are not strong acids like those shown to be required to promote PDeB. ${ }^{16-19}$ The neutral pathway proposed by Kuivila also relies upon their acidity, which requires addition of water with liberation of a proton, and is highly unlikely under neutral conditions. ${ }^{18}$

The first decomposition pathway considered by ourselves was that PDeB of neat boronic acids occurs upon long-term exposure to atmospheric water during storage. Computationally, two mechanisms were found. The first involves one water molecule and a one-step process; the water molecule provides a proton to the aromatic carbon atom while simultaneously forming an O-B bond and cleaving the $\mathrm{C}-\mathrm{B}$ bond. The transition state for this process, TS1, is shown in Fig. 1 and corresponds to a free energy barrier of $47.0 \mathrm{kcal} \mathrm{mol}{ }^{-1}$. All calculations employed the M06/6-311+G**//B3LYP/6-31+G** level of theory with PCM solvation and were performed in Gaussian09 and 03. ${ }^{20-27}$ Benchmarking calculations revealed that this level of theory performs well compared to MP2/ $6-311+G^{* *}$ calculations (see ESI $\dagger$ ). Plumley and Evanseck have shown that M06-2X performs better than M06 for borane-
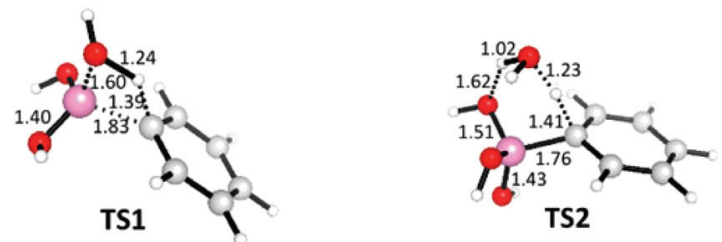

Fig. 1 Two transition states for PDeB of phenyl boronic acid by water. Distances given in $\AA$. Boron atoms are shown in pink. 
ammonia complexes but Lee et al. selected M06 as a more accurate level of theory, in line with our own findings. ${ }^{28-31}$

A six-membered ring transition state alternative, in which a second water molecule facilitates the reaction, was also located and the lowest energy possibility is shown as TS2 in Fig. 1. Structure TS2 corresponds to a gas phase enthalpy of activation $\left(H_{\text {act }}\right)$ of $19.8 \mathrm{kcal} \mathrm{mol}^{-1}$ that is lower than TS1, which has a computed $H_{\text {act }}$ of $34.0 \mathrm{kcal} \mathrm{mol}^{-1}$. However, the entropic penalty involved in bringing three species together in TS2 results in an aqueous phase free energy barrier that is $3.3 \mathrm{kcal}$ $\mathrm{mol}^{-1}$ higher than that for TS1. Despite the errors inherent to the calculations, these barriers are inconsistent with degradation over the course of days to years. The observation of Hall, that water might actually stabilise boronic acids is, at the least, not impossible. ${ }^{32}$ The measurements of half lives and activation free energies for a range of biologically important reactions in water by Wolfenden and co-workers (plotted in ESI $\dagger$ ) suggest that degradation over the course of days to years would be consistent with free energy barriers in the 27-33 kcal mol $^{-1}$ range. $^{33}$

Three examples were selected for experimental study in order to verify this surprising finding (i.e. that water is not the causative agent in PDeB): furan-2-yl-, thiophen-2-yl- and benzofuran-2-yl-boronic acid, each of which have been reported to degrade significantly within 15 days when stored as neat reagents. ${ }^{9}$ When heated under microwave irradiation to $130{ }^{\circ} \mathrm{C}$ for 30 minutes, in either water alone or in tetrahydrofuran : water (4:1) mixtures (to aid solubility of the boronic acid), little or no degradation could be observed when the reaction mixtures were analyzed by LCMS (see ESI $\dagger$ ). The lack of decomposition of furan-2-yl boronic acid under these conditions is especially surprising because this is reported to be one of the more unstable heterocyclic boronic acids. ${ }^{9}$ Experiment and theory concur that water alone is not responsible for the PDeB of boronic acids upon storage.

Attention was next turned to the boronic acid molecules themselves. Transition states analogous to TS1 but where a boronic acid provides both the Lewis basic oxygen atom and the requisite proton were computed: the lowest enthalpy example is TS3. The free energy barriers for these processes (Fig. 2) are also prohibitively high, with the lowest being over $50 \mathrm{kcal} \mathrm{mol}^{-1}$. If, however, there were a reduced or eliminated entropic barrier, the enthalpic barrier (34.1 $\left.\mathrm{kcal} \mathrm{mol}^{-1}\right)$ would be in line with the free energy barrier for a process that occurs at room temperature over the course of days to years.

During formation of the solid state, various crystal packing forces determine the arrangement of the functional groups and might pre-organize boronic acids for $\mathrm{PDeB}$, thereby reducing the entropic cost of bringing two molecules together. All of the crystal structures available for boronic acids published in the Cambridge crystallographic database (CCD) were retrieved and several geometrical parameters, describing the position of each boronic acid relative to its neighbors, were measured (Fig. 3). ${ }^{34}$

The distribution of boron to oxygen distances, $d$, is shown in Fig. 3(A). The number of structures with $d$ less than $4 \AA$ is
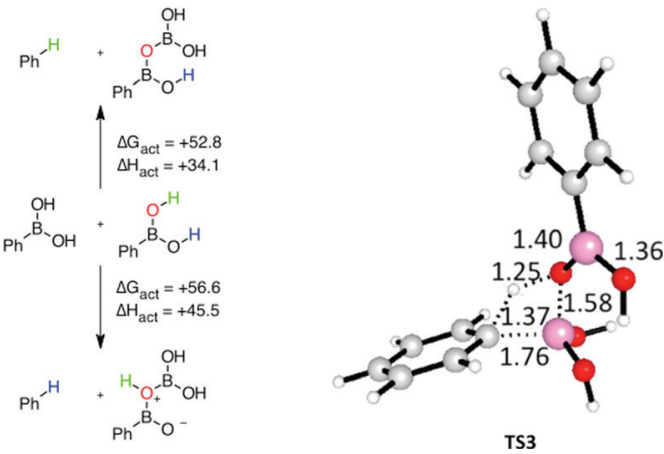

Fig. 2 Energetics for boronic acid mediated PDeB. Free energy and enthalpy changes are comparisons with two molecules of boronic acid and are in $\mathrm{kcal} \mathrm{mol}^{-1}$. Distances given in $\AA$. Boron atoms are shown in pink.

enhanced and those with $d$ less than $3.7 \AA$ are shown as blue crosses in Fig. 3(B). The plot of $\theta_{1}$ vs. $\theta_{2}$ shown in Fig. 3(B), identifies commonly occurring supramolecular structural features. The area highlighted by the purple box corresponds to the cyclic hydrogen bonding interaction shown below the plot. Hall stated that boronic acids regularly form these interactions in the solid state. ${ }^{32}$ The orange box in Fig. 3(B) corresponds to an interaction between the oxygen lone pair of one boronic acid and the Lewis-acidic boron atom of another (assuming lone pairs in the $89-140^{\circ}$ positions on the oxygen atoms) and represents examples 'primed for PDeB'. It is found that 16 of the $74(21.6 \%)$ crystal structures that have $d<3.7 \AA$ satisfy these criteria. The crystal structure of phenylboronic acid illustrates the key solid-state structural motifs (end-to-end hydrogen bonding and boron-oxygen interaction) for boronic acids and is shown in Fig. $4 .^{35}$ Further crystal structure analysis is presented in the ESI. $\dagger$ The manuscripts describing the preparation of material leading to the crystal structures represented by the blue crosses in the orange box were examined. The yield of the compound leading to this crystal structure was compared to that of all other boronic acids described in the same paper. ${ }^{35-54}$ Such compounds were prepared under similar conditions and so their yields can reasonably be compared. It was found (see ESI $\dagger$ ) that compounds which form crystal structures that satisfy the geometric requirements highlighted in Fig. 3 are formed in $11 \%$ lower yield on average than other boronic acids reported in the same paper. Given that compounds are presumably prepared in the solid state and studied crystallographically soon after and that the other compounds in the papers remain uncharacterized by crystallography and so may also provide the correct geometries, it is at least suggestive that this geometrically identified subset is prone to faster degradation. Although the pre-organization of boronic acids in the solid state can promote the $\mathrm{PDeB}$ reaction, arrangements that place boronic acids in geometries that are not primed for PDeB (the majority) are likely to protect them from degradation. These studies have examined crystalline solids, but the same motifs most likely dominate in amorphous solids which will also include a range of orientations and retain limited mobility. ${ }^{55,56}$ 


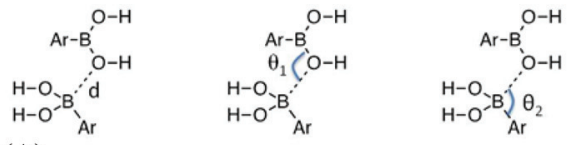

(A)

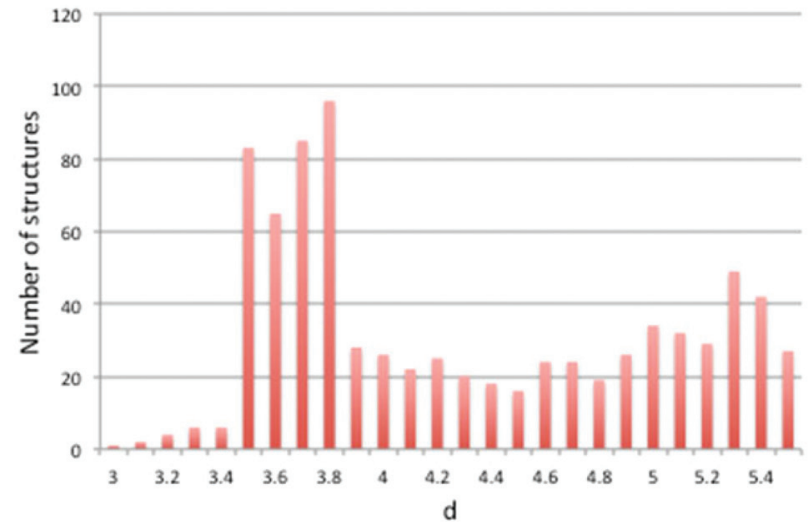

(B)

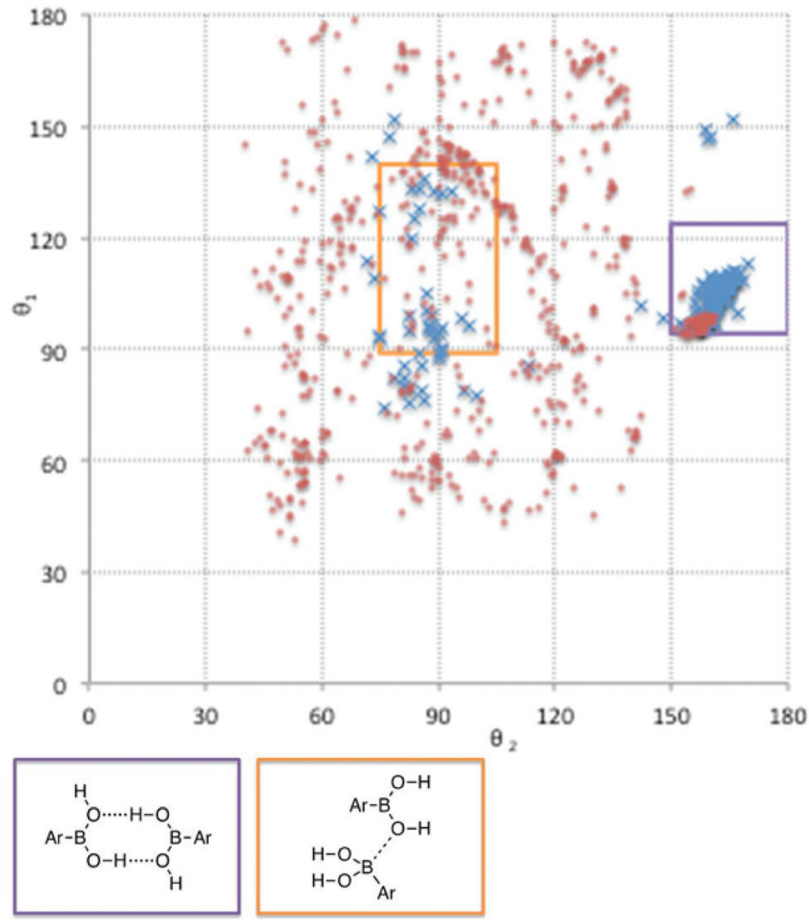

Fig. 3 Geometrical analysis of boronic acids in solid state structures in the CCD. (A) The frequency of occurrence of $B$ to $O$ distance $d$ and (B) the observed combinations of $\theta_{1}$ and $\theta_{2}$. Structures with $d<3.7 \AA$ are highlighted as blue crosses.

The consequences of the end-on-end hydrogen bonding interaction were probed by computing energetics in its presence; these are compared to those in its absence in Fig. 5. There is a substantial $\left(9.6 \mathrm{kcal} \mathrm{mol}^{-1}\right)$ driving force for the formation of the hydrogen bond which, although overstated in gas phase calculations, is consistent with the observations shown in Fig. 3. The compromises involved in forming the solid state should ensure that examples which are primed for PDeB have an energy in the region highlighted in purple in

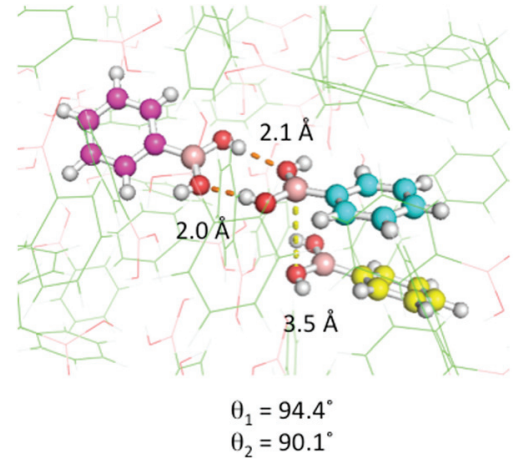

Fig. 4 Crystal structure of $\mathrm{Ph}-\mathrm{B}(\mathrm{OH})_{2}$. The carbons in three molecules of $\mathrm{Ph}-\mathrm{B}(\mathrm{OH})_{2}$ are shown as purple, cyan and yellow spheres while the lattice is shown as green lines.

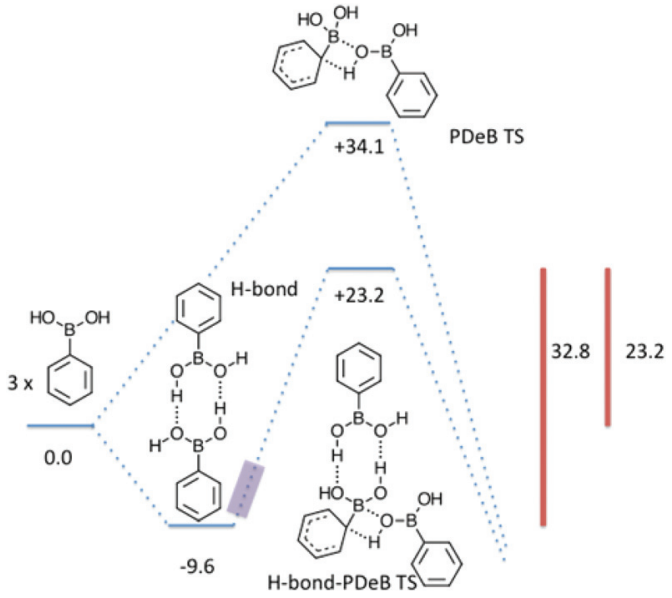

Fig. 5 The effect of intermolecular hydrogen bonds on boronic acid mediated PDeB. Gas phase enthalpies in $\mathrm{kcal} \mathrm{mol}^{-1}$ relative to three molecules of boronic acid are provided.

Table 1 Energetics of PDeB for some unstable boronic acids. ${ }^{10}$ All enthalpies in $\mathrm{kcal} \mathrm{mol}^{-1}$ are relative to those for three molecules of boronic acid

\begin{tabular}{lrlrl}
\hline & $\begin{array}{l}\text { \% Boronic acid } \\
\text { remaining }^{10}\end{array}$ & $\begin{array}{l}H_{\text {rel }} \\
\text { PDeB TS }\end{array}$ & $\begin{array}{l}H_{\text {rel }} \\
\text { H-Bond }\end{array}$ & $\begin{array}{l}H_{\text {rel H-bond- }} \\
\text { PDeB TS }\end{array}$ \\
\hline Phenyl & na & 34.1 & -9.6 & 23.2 \\
2-Furanyl & 7 & 34.8 & -10.0 & 23.8 \\
2-Benzofuranyl & 88 & 35.9 & -10.1 & 24.8 \\
2-Thiophenyl & 80 & 31.2 & -8.5 & 21.0 \\
Vinyl & 5 & 34.0 & -9.5 & 23.7 \\
Cyclopropyl & 31 & 33.9 & -9.6 & 23.2 \\
& & & &
\end{tabular}

Fig. 5, in which the full enthalpy of stabilization due to hydrogen bonding is not achieved and in which the O-B interaction is partially formed. Such a structure is substantially arranged as required for the $\mathrm{PDeB}$ process and the enthalpic barrier must therefore be at least $23.2 \mathrm{kcal}^{-1}$ but less than $32.8 \mathrm{kcal} \mathrm{mol}^{-1}$ for phenylboronic acid. Such a value, com- 


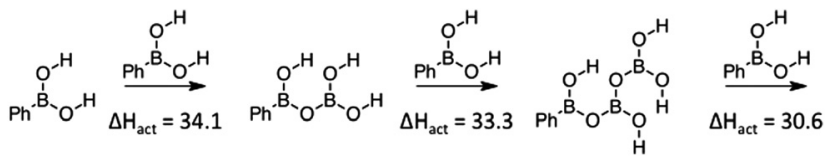

Fig. 6 PDeB involving the products formed in boronic acid mediated PDeB. The activation enthalpy for individual steps are provided in $\mathrm{kcal} \mathrm{mol}^{-1}$.

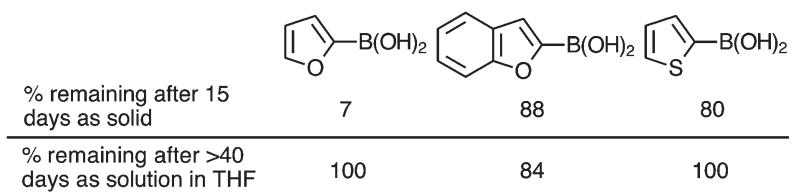

Fig. 7 Contrasting behavior of boronic acids in the solid state and in THF solution.

bined with a modest $T \Delta S$ component, is entirely consistent with degradation over the course of days to years.

The enthalpic barriers have been computed for the processes shown in Fig. 5 for some of the examples found by Burke and co-workers to degrade over the course of 15 days upon benchtop storage (Table 1). ${ }^{9}$ The observed levels of degradation are consistent with the magnitude of the computed barriers.

This process for PDeB may be further enhanced because the product of the reaction is computed to be more reactive than the reactant. As summarized in Fig. 6, with each reaction, a larger agglomeration of boronic anhydrides could build up which have enhanced Lewis acidity and are more prone to react with a molecule of boronic acid than their precursor. This would suggest that the appropriately structured crystalline material could degrade very rapidly because each step would create a more reactive molecule and be positioned ready to react. This must be set against an unknown effect upon the entropy of activation. Further investigations would be required to investigate whether this computed effect is manifested experimentally. The six-membered boroxine ring is also computed to be more reactive but to be an energetically disfavored species and we were unable to find evidence of them playing a significant role in PDeB of neat boronic acids.

The proposed mechanism would imply that unstable boronic acids could be stored in solution. This was found to be the case and boronic acids previously shown to be unstable as neat reagents, over the course of 15 days, undergo little or no decomposition after 1-2 months in THF solution (Fig. 7), even using reagent-grade solvent that is neither dried nor degassed. As well as being of practical relevance to the storage and utility of these reagents, these observations also provide support for a 'solid-state' promoted decomposition pathway.

In conclusion, we have carried out a practical and computational study of the possible mechanistic pathways for the PDeB of boronic acids when stored as neat reagents. Prohibitively high computed free-energy barriers for the decomposition of boronic acids by water, together with the practical observation that little PDeB occurs in the presence of excess water suggest that water alone is not the causative agent in PDeB of neat boronic acid samples. We propose a solid-state pre-organization effect, which is backed by the presence of the required supramolecular arrangements in the CCD, as well as by the fact that little or no decomposition is observed for notoriously unstable boronic acids e.g. furan-2-ylboronic acid, when stored in solution for prolonged periods. Computed enthalpies of reaction combined with small entropic barriers, as expected for pre-arranged species, would entail a free energy barrier consistent with the observed rate of degradation. We recommend that unstable boronic acids be stored as solutions whenever they are not required for immediate use.

\section{Acknowledgements}

This research was carried out as part of the AstraZeneca global post-doctoral scheme and the authors would like to acknowledge the scientific freedom provided by AstraZeneca throughout the work. Special thanks are due to Dr Jennifer Pink (AstraZeneca) for many useful discussions throughout this research. The authors would also like to acknowledge the use of the EPSRC UK National Service for Computational Chemistry Software (NSCCS) at Imperial College London in carrying out this work.

\section{Notes and references}

1 A. Fersht, in Structure and Mechanism in Protein Science, W. H. Freeman and Co., New York, 1999.

2 M. I. Page and W. P. Jencks, Entropic contributions to rate accelerations in enzymic and intramolecular reactions and the chelate effect, Proc. Natl. Acad. Sci. U. S. A., 1971, 68, 1678-1683.

3 S. P. Kim, A. G. Leach and K. N. Houk, The Origins of Noncovalent Catalysis of Intermolecular Diels-Alder Reactions by Cyclodextrins, Self-Assembling Capsules, Antibodies, and RNAses, J. Org. Chem., 2002, 67, 4250-4260.

$4 \mathrm{~J}$. Villa, et al., How important are entropic contributions to enzyme catalysis?, Proc. Natl. Acad. Sci. U. S. A., 2000, 97, 11899-11904.

5 A. d. Meijere and F. Diederich, in Metal-catalyzed crosscoupling reactions, ed. A. de Meijere and F. Diederich, Wiley-VCH, Weinheim, Chichester, 2004.

6 C. C. Johansson Seechurn, M. O. Kitching, T. J. Colacot and V. Snieckus, Palladium-catalyzed cross-coupling: a historical contextual perspective to the 2010 Nobel Prize, Angew. Chem., Int. Ed., 2012, 51, 5062-5085.

7 D. G. Hall, in Boronic acids: volume 1: preparation and applications in organic synthesis, medicine and materials, Wiley-VCH, Weinheim, 2011.

8 S. Kotha, K. Lahiri and D. Kashinath, Recent applications of the Suzuki-Miyaura cross-coupling reaction in organic synthesis, Tetrahedron, 2002, 58, 9633-9695. 
9 D. M. Knapp, E. P. Gillis and M. D. Burke, A General Solution for Unstable Boronic Acids: Slow-Release CrossCoupling from Air-Stable MIDA Boronates, J. Am. Chem. Soc., 2009, 131, 6961-6963.

10 S. Darses and J. P. Genet, Potassium organotrifluoroborates: new perspectives in organic synthesis, Chem. Rev., 2008, 108, 288-325.

11 E. Vedejs, R. Chapman, S. Fields, S. Lin and M. Schrimpf, Conversion of arylboronic acids into potassium aryltrifluoroborates: Convenient precursors of arylboron difluoride lewis acids, J. Org. Chem., 1995, 60, 3020-3027.

12 G. A. Molander and B. Canturk, Organotrifluoroborates and monocoordinated palladium complexes as catalysts-a perfect combination for Suzuki-Miyaura coupling, Angew. Chem., Int. Ed., 2009, 48, 9240-9261.

13 M. Butters, et al., Aryl trifluoroborates in Suzuki-Miyaura coupling: the roles of endogenous aryl boronic acid and fluoride, Angew. Chem., Int. Ed., 2010, 49, 5156-5160.

14 A. J. J. Lennox and G. C. Lloyd-Jones, The slow-release strategy in Suzuki-Miyaura coupling, Isr. J. Chem., 2010, 50, 664-674.

15 A. J. Lennox and G. C. Lloyd-Jones, Organotrifluoroborate Hydrolysis: Boronic Acid Release Mechanism and an AcidBase Paradox in Cross-Coupling, J. Am. Chem. Soc., 2012, 134, 7431-7441.

16 H. G. Kuivila and K. V. Nahabedian, Electrophilic Displacement Reactions. XI. Solvent Isotope Effects in the Protodeboronation of Areneboronic Acids1-3, J. Am. Chem. Soc., 1961, 83, 2164-2166.

17 H. G. Kuivila and K. V. Nahabedian, Electrophilic Displacement Reactions. X. General Acid Catalysis in the Protodeboronation of Areneboronic Acids1-3, J. Am. Chem. Soc., 1961, 83, 2159-2163.

18 H. G. Kuivila, J. F. Reuwer Jr. and J. A. Mangravite, Electrophilic displacement reactions: XV. Kinetics and mechanism of the base-catalyzed protodeboronation of areneboronic acids, Can. J. Chem., 1963, 41, 3081-3090.

19 K. V. Nahabedian and H. G. Kuivila, Electrophilic Displacement Reactions. XII. Substituent Effects in the Protodeboronation of Areneboronic Acids1-3, J. Am. Chem. Soc., 1961, 83, 2167-2174.

20 Y. Zhao and D. G. Truhlar, The M06 suite of density functionals for main group thermochemistry, thermochemical kinetics, noncovalent interactions, excited states, and transition elements: two new functionals and systematic testing of four M06-class functionals and 12 other functionals, Theor. Chem. Acc., 2008, 120, 215-241.

21 Y. Zhao and D. G. Truhlar, Density Functionals with Broad Applicability in Chemistry, Acc. Chem. Res., 2008, 41, 157167.

22 A. D. Becke, Density-functional thermochemistry. III. The role of exact exchange, J. Chem. Phys., 1993, 98, 5648-5652.

23 C. Lee, W. Yang and R. G. Parr, Development of the ColleSalvetti correlation-energy formula into a functional of the electron density, Phys. Rev. B: Condens. Matter, 1988, 37, 785-789.
24 P. C. Hariharan and J. A. Pople, Influence of polarization functions on MO hydrogenation energies, Theor. Chim. Acta, 1973, 28, 213-222.

25 J. Tomasi, B. Mennucci and E. Cances, The IEF version of the PCM solvation method: an overview of a new method addressed to study molecular solutes at the QM ab initio level, J. Mol. Struct. (THEOCHEM), 1999, 464, 211-226.

26 M. J. Frisch, et al., Gaussian 09 (Revision A.1).

27 M. J. Frisch, G. W. Trucks, H. B. Schlegel, G. E. Scuseria, M. A. Robb, J. R. Cheeseman, J. A. Montgomery Jr., T. Vreven, K. N. Kudin, J. C. Burant, J. M. Millam, S. S. Iyengar, J. Tomasi, V. Barone, B. Mennucci, M. Cossi, G. Scalmani, N. Rega, G. A. Petersson, H. Nakatsuji, M. Hada, M. Ehara, K. Toyota, R. Fukuda, J. Hasegawa, M. Ishida, T. Nakajima, Y. Honda, O. Kitao, H. Nakai, M. Klene, X. Li, J. E. Knox, H. P. Hratchian, J. B. Cross, V. Bakken, C. Adamo, J. Jaramillo, R. Gomperts, R. E. Stratmann, O. Yazyev, A. J. Austin, R. Cammi, C. Pomelli, J. W. Ochterski, P. Y. Ayala, K. Morokuma, G. A. Voth, P. Salvador, J. J. Dannenberg, V. G. Zakrzewski, S. Dapprich, A. D. Daniels, M. C. Strain, O. Farkas, D. K. Malick, A. D. Rabuck, K. Raghavachari, J. B. Foresman, J. V. Ortiz, Q. Cui, A. G. Baboul, S. Clifford, J. Cioslowski, B. B. Stefanov, G. Liu, A. Liashenko, P. Piskorz, I. Komaromi, R. L. Martin, D. J. Fox, T. Keith, M. A. Al-Laham, C. Y. Peng, A. Nanayakkara, M. Challacombe, P. M. W. Gill, B. Johnson, W. Chen, M. W. Wong, C. Gonzalez and J. A. Pople, Gaussian 03 (Revision C.02), Gaussian, Inc., Wallingford CT, 2004.

28 J. A. Plumley and J. D. Evanseck, Covalent and ionic nature of the dative bond and account of accurate ammonia borane binding enthalpies, J. Phys. Chem. A, 2007, 111, 13472-13483.

29 J. A. Plumley and J. D. Evanseck, Hybrid Meta-Generalized Gradient Functional Modeling of Boron-Nitrogen Coordinate Covalent Bonds, J. Chem. Theory Comput., 2008, 4, 1249-1253.

30 J. A. Plumley and J. D. Evanseck, Periodic Trends and Index of Boron Lewis Acidity, J. Phys. Chem. A, 2009, 113, 59855992.

31 J. M. Lee, P. Helquist and O. Wiest, Diastereoselectivity in Lewis-Acid-Catalyzed Mukaiyama Aldol Reactions: A DFT Study, J. Am. Chem. Soc., 2012, 134, 14973-14981.

32 D. G. Hall, in Boronic acids: preparation and applications in organic synthesis and medicine, Wiley-VCH, John Wiley, Weinheim, Chichester, 2005.

33 R. Wolfenden, Benchmark reaction rates, the stability of biological molecules in water, and the evolution of catalytic power in enzymes, Annu. Rev. Biochem., 2011, 80, 645667.

34 F. H. Allen, The Cambridge Structural Database: a quarter of a million crystal structures and rising, Acta Crystallogr., Sect. B: Struct. Sci., 2002, 58, 380-388.

35 S. J. Rettig and J. Trotter, Crystal and molecular structure of phenylboronic acid, C6H5B(OH)2, Can. J. Chem., 1977, 55, 3071-3075. 
36 C. Zheng, B. Spielvogel, R. Smith and N. Hosmane, Crystal structure of 4-methylphenylboronic acid, C7H9BO2, Z. Kristallogr. - New Cryst. Struct., 2001, 216, 363-364.

37 N. Saygili, A. S. Batsanov and M. R. Bryce, 5-Pyrimidylboronic acid and 2-methoxy-5-pyrimidylboronic acid: new heteroarylpyrimidine derivatives via Suzuki cross-coupling reactions, Org. Biomol. Chem., 2004, 2, 852-857.

38 P. Rodríguez-Cuamatzi, G. Vargas-Díaz and H. Höpfl, Modification of $2 \mathrm{D}$ water that contains hexameric units in chair and boat conformations-a contribution to the structural elucidation of bulk water, Angew. Chem., Int. Ed., 2004, 116, 3103-3106.

39 K. M. Clapham, et al., New Pyrimidylboronic Acids and Functionalized Heteroarylpyrimidines by Suzuki CrossCoupling Reactions, Eur. J. Org. Chem., 2007, 5712-5716.

40 A. E. Smith, K. M. Clapham, A. S. Batsanov, M. R. Bryce and B. Tarbit, (Dimethoxy-and Dihalopyridyl) boronic Acids and Highly Functionalized Heteroarylpyridines by Suzuki Cross-Coupling Reactions, Eur. J. Org. Chem., 2008, 1458-1463.

41 K. M. Clapham, A. S. Batsanov, M. R. Bryce and B. Tarbit, Trifluoromethyl-substituted pyridyl- and pyrazolylboronic acids and esters: synthesis and Suzuki-Miyaura crosscoupling reactions, Org. Biomol. Chem., 2009, 7, 21552161.

42 M. Dabrowski, S. Lulinski, J. Serwatowski and M. Szczerbinska, (2-Methoxy-3-pyridyl) boronic acid, Acta Crystallogr., Sect. C: Cryst. Struct. Commun., 2006, 62, o702o704.

43 K. Kacprzak, T. Klis and J. Serwatowski, [3-Bromo-2-(3fluorobenzyloxy) phenyl] boronic acid, Acta Crystallogr., Sect. E: Struct. Rep. Online, 2009, 65, o2250-02250.

44 M. R. Shimpi, N. SeethaLekshmi and V. R. Pedireddi, Supramolecular architecture in some 4-halophenylboronic acids, Cryst. Growth Des., 2007, 7, 1958-1963.

45 S. Das, V. L. Alexeev, A. C. Sharma, S. J. Geib and S. A. Asher, Synthesis and crystal structure of 4-amino3-fluorophenylboronic acid, Tetrahedron Lett., 2003, 44, 7719-7722.
46 C. B. Aakeröy, J. Desper, B. Levin and D. J. Salmon, Boronic Acids As Versatile Supramolecular Reagents, ACA Trans., 2004, 39, 123-129.

47 M. K. Cyrański, A. Jezierska, P. Klimentowska, J. J. Panek and A. Sporzyński, Impact of intermolecular hydrogen bond on structural properties of phenylboronic acid: quantum chemical and X-ray study, J. Phys. Org. Chem., 2008, 21, 472-482.

48 R. M. Al-Zoubi and D. G. Hall, Mild silver (I)-mediated regioselective iodination and bromination of arylboronic acids, Org. Lett., 2010, 12, 2480-2483.

49 J. Serwatowski, T. Klis and K. Kacprzak, 3-Bromo-2-(2fluorobenzyloxy) phenylboronic acid, Acta Crystallogr., Sect. E: Struct. Rep. Online, 2006, 62, o1308-01309.

50 R. Sarma and J. B. Baruah, B $\cdots \pi$-aromatic and $\mathrm{C}-\mathrm{H} \cdots \mathrm{B}$ interactions in co-crystals of aromatic amine $\mathrm{N}$-oxides with p-phenylenediboronic acid, J. Mol. Struct., 2009, 920, 350354.

51 A. E. Thompson, et al., 2-Ethoxy-3-pyridylboronic acid: a versatile reagent for the synthesis of highly-functionalised 3-aryl/heteroaryl-pyridines via Suzuki cross-coupling reactions, Tetrahedron, 2005, 61, 5131-5135.

52 S. Lulinski and J. Serwatowski, 2-(Methoxycarbonyl) phenylboronic acid, Acta Crystallogr., Sect. C: Cryst. Struct. Commun., 2006, 62, o301-0303.

53 M. Filthaus, I. M. Oppel and H. F. Bettinger, Supramolecular structures and spontaneous resolution: the case of ortho-substituted phenylboronic acids, Org. Biomol. Chem., 2008, 6, 1201-1207.

54 M. Dabrowski, S. Lulinski and J. Serwatowski, (2-Methoxy1, 3-phenylene) diboronic acid, Acta Crystallogr., Sect. E: Struct. Rep. Online, 2008, 64, o414-0415.

55 L. Yu, Amorphous pharmaceutical solids: preparation, characterization and stabilization, Adv. Drug Delivery Rev., 2001, 48, 27-42.

56 M. C. Wilding, M. Wilson and P. F. McMillan, Structural studies and polymorphism in amorphous solids and liquids at high pressure, Chem. Soc. Rev., 2006, 35, 964986. 